\title{
Multiparticle biased diffusion-limited aggregation with surface diffusion: A comprehensive model of electrodeposition
}

\author{
Mario Castro, ${ }^{1,2}$ Rodolfo Cuerno, ${ }^{3}$ Angel Sánchez, ${ }^{3}$ and Francisco Domínguez-Adame ${ }^{2}$ \\ ${ }^{1}$ Universidad Pontificia de Comillas, E-28015 Madrid, Spain \\ ${ }^{2}$ Grupo Interdisciplinar de Sistemas Complicados, Departamento de Física de Materiales, Facultad de Ciencias Físicas, \\ Universidad Complutense, E-28040 Madrid, Spain \\ ${ }^{3}$ Grupo Interdisciplinar de Sistemas Complicados, Departamento de Matemáticas, Universidad Carlos III de Madrid, \\ E-28911 Leganés, Madrid, Spain \\ (Received 10 December 1999)
}

\begin{abstract}
We present a complete study of the multiparticle biased diffusion-limited aggregation (MBDLA) model supplemented with surface diffusion (SD), focusing on the relevance and effects of the latter transport mechanism. By comparing different algorithms, we show that MBDLA + SD is a very good qualitative model for electrodeposition in essentially the whole range of current intensities provided one introduces SD in the model in the proper fashion. We have found that the correct procedure involves simultaneous bulk diffusion and SD, introducing a time scale arising from the ratio of the rates of the two processes. We discuss in detail the different morphologies obtained and compare them to the available experimental data with very satisfactory results. We also characterize the aggregates thus obtained by means of the dynamic scaling exponents of the interface height, allowing us to distinguish several regimes in the mentioned interface growth. Our asymptotic scaling exponents are again in good agreement with recent experiments. We conclude by discussing a global picture of the influence and consequences of SD in electrodeposition.
\end{abstract}

PACS number(s): 05.40.-a, 05.70.Ln, 68.35.Fx, 81.15.Pq

\section{INTRODUCTION}

Quasi-two-dimensional (quasi-2D) electrochemical deposition (ECD) [1-9] has become one of the most widely studied pattern forming processes since its recognition as a paradigm of nonlocal, nonequilibrium growth processes [1,2]. Within this general context, a great deal of work has been devoted in the past fifteen years to experimental and theoretical studies of quasi-2D ECD. A first group of work deals mainly with pattern formation, its main results concerning "phase diagrams" of morphologies [10,11], ECD as a Laplacian growth process [12-16], dynamic morphological transitions $[17,18]$, etc. All these studies aim to understand the principles underlying the rich variety of morphologies observed, ranging from dendritic to fractal. In addition to this line of research, there is a second one [19-24] whose main interest is the existence of universality and scale invariance in the roughness of the deposits produced [1,2]. From all this and related research, it is now believed that complex structures with different morphologies arise from quasi-2D ECD due to the interplay of different transport mechanisms, such as cation diffusion, electromigration, fluid convection, and surface diffusion [3-9]. However, the combined effect of all these factors leads to a very complex process, and it is becoming increasingly apparent that ECD is not well understood yet. In particular, the detailed role of surface diffusion (SD) is still an open question that hinders our understanding of both the morphologies and the scaling of ECD aggregates.

Much of the work mentioned in the above paragraph has been motivated by the quest to find a universal model to help understand ECD phenomena. The first model formulated with that purpose was the famous computer algorithm known as diffusion limited aggregation (DLA) [12], in which a par- ticle diffuses on a lattice and attaches to the growing aggregate at the place where it first hits. It is not difficult to observe (see [1,2] and references therein) that this simple model represents the zero concentration, quasistatic limit of ECD. Therefore, its validity as a general description of ECD is rather restricted because it does not include most of the effects involved in the process. However, DLA has played a seminal role as a source of inspiration both for continuum approaches [25-27] — which predict some high-current properties but take into account neither the influence of the applied voltage nor the electrolyte concentration-and for more sophisticated computer models, basically modifications of DLA (see, e.g., [28-30] and also the paragraph below), which are more or less phenomenological and concentrate on changes in morphology, thus being unable to explain the underlying mechanisms yielding those patterns.

In this paper, we report the results of detailed numerical studies of multiparticle biased diffusion-limited aggregation (MBDLA) [16,23] supplemented with SD. MBDLA is a model in the family of multiparticle DLA models [1,30-33], in which a finite number of random walkers, possibly with constant concentration, is introduced instead of the single walker of DLA. Thus, the excluded volume interaction among the walkers leads to several of the effects neglected in DLA. As its main ingredient, MBDLA includes, in addition, a preferential bias (which had been first studied in the context of single-particle, DLA models by Meakin [34]) of the walkers toward the cathode to mimic the electric field: In this form, the model was successfully introduced in [16] to study the influence of the applied electric field on the composition of magnetic, amorphous CoP alloys grown by ECD at constant current. The main virtue of MBDLA is that it is a mesoscopic model embedded on a two-dimensional square lattice, but it reproduces the mean fractions of $\mathrm{Co}$ and $\mathrm{P}$ in 
two-cation species ECD, as well as the qualitative morphology of the product electrodeposits. In fact, the agreement between MBDLA and ECD experiments [35] is quantitative, as the electrical current intensity and the experiment time can be directly related to the simulation parameters [16]. Therefore, we are confident that MBDLA is a good starting point to study the relevance of SD in ECD and, specifically, its influence on the shape of the aggregates and their dynamic scaling. Scaling properties of MBDLA without SD were briefly reported in [23].

The report of our results is organized as follows. We describe our model in Sec. II, where a brief introduction to the physics and chemistry of ECD is followed by a detailed account of the rules governing MBDLA. Section III reports our numerical results, such as morphological patterns and roughness scaling. After physically showing that SD has to be included, we introduce three different rules for SD, which are carefully considered and compared to experiments, allowing us to identify the proper way to introduce SD in the model. Finally, we conclude in Sec. IV with a discussion of our results, which will allow us to suggest a reasonably approximate picture of ECD phenomena. A few technical details about one of the rules for SD are given in an Appendix.

\section{THE MODEL}

\section{A. Basic facts about ECD}

Prior to describing in detail what MBDLA is, and in order to motivate and to better understand the model rules, we will briefly summarize the basic physics and chemistry of ECD, by collecting the equations commonly accepted to govern its main features (see, e.g., [5,6] for further details). Generally speaking, ECD experiments involve two species, named cations and anions, moving in an incompressible viscous fluid. In very many cases, ECD takes place in quasi-2D cells with parallel electrodes. The cations move toward the cathode and the anions toward the anode. The basic equations for the concentrations of both species are as follows:

$$
\begin{gathered}
\frac{\partial C}{\partial t}=-\boldsymbol{\nabla} \cdot \mathbf{J}_{c}, \\
\frac{\partial A}{\partial t}=-\boldsymbol{\nabla} \cdot \mathbf{J}_{a}, \\
\mathbf{J}_{c}=-D_{c} \boldsymbol{\nabla} C+\mu_{c} \mathbf{E} C+\mathbf{v} C, \\
\mathbf{J}_{a}=-D_{a} \boldsymbol{\nabla} A-\mu_{a} \mathbf{E} A+\mathbf{v} A,
\end{gathered}
$$

where $C$ and $A$ are the cation and anion concentrations, respectively, $D_{c, a}$ the cationic and anionic diffusion coefficients, $\mu_{c, a}$ their mobilities, $\mathbf{v}$ the fluid velocity field, and $\mathbf{E}$ the electric field along the cell. The latter is related to cation and anion concentrations via the Poisson equation

$$
\boldsymbol{\nabla} \cdot \mathbf{E}=-\nabla^{2} \phi=-e\left(z_{c} C-z_{a} A\right) / \varepsilon,
$$

where $\phi$ is the applied potencial, $e z_{c}$ and $-e z_{a}$ are the cation and anion electric charges, respectively, and $\varepsilon$ is the dielectric permittivity of the fluid. Generally speaking, matter balance across the interface leads to an interface velocity proportional to the flux of cations and therefore, in the absence of any other limiting process, proportional to the current density as well. In addition, except for the region close to the cathode, we may assume electroneutrality [5], which in turn implies that the cation mean velocity is constant. The incompressible Navier-Stokes equations determine the velocity $\mathbf{v}$ of the solvent. Fluid convection is always present in ECD experiments, but in many instances it can be small enough to be safely neglected, as has been shown in actual experiments [36,37].

When the cations arrive at the cathode, they are reduced irreversibly and an aggregate of neutral particles begins to grow. The particles on the surface aggregate are transported along it due to local chemical potential gradients. The resulting particle current conserves the number of particles on the surface and is given approximately by (see [38] for a detailed discussion)

$$
\mathbf{J}_{s} \propto-\nabla_{s} \kappa,
$$

where $\mathbf{J}_{s}$ is the particle current along the surface, $\kappa$ the interface local curvature at each site, and $\boldsymbol{\nabla}_{s}$ the gradient taken along the surface. Roughly speaking, SD tends to reduce the interface local curvature. Finally, we note that the mean concentration of charge carriers in the bath is constant as new cations are formed at the anode upon arrival of the anions [7].

\section{B. Definition and rules of MBDLA}

In this section we will define MBDLA through its evolution rules, for which we take into account the physical equations presented in the previous section. At this point, we do not consider SD, whose need will be justified in the next section, and consequently we postpone the discussion of the rules to implement SD as well. Thus, MBDLA is a cellular automaton defined on a two-dimensional square lattice of horizontal dimension $L_{x}$ and vertical dimension $L_{y}$ (with lateral periodic boundary conditions and reflective boundary condition at the top; for the conditions at the bottom, see below), in which a number of random walkers (cations) are randomly distributed with concentration $c$. The bottom of the lattice is chosen to be the cathode. We do not consider the anion dynamics, but we implicitly introduce it by the creation of particles and by charge electroneutrality [39].

The initial condition evolves in time as follows. At every time step a walker is chosen and moved to one of its four neighboring sites with probabilities taken from a finite difference scheme of Eqs. (1a) and (1c) [40]: probability 1/(4 $+p)$ to move either left, right, or upward, and probability $(1+p) /(4+p)$ to move down, i.e., toward the cathode. The parameter $p$ is referred to as the bias; in galvanostatic conditions it can be quantitatively related to the electric current density in the physical system as shown in [16]. Let us stress here that our present choice for the probabilities is different from that reported in [16] and [23], but we have checked that the results hardly differ from those presented in this paper. The main reason for this new selection is that, with the new rules, the bias $p$ ranges from 0 to $\infty$, that is, from pure multiparticle DLA to ballistic deposition, whereas the rules in the mentioned references allow for a range in $p$ from 0 to 0.25 , and the ballistic deposition limit cannot be reached 
(although $p=0.25$ already; is rather close, see [16,23]). After a destination site has been chosen, the particle moves if that node of the lattice is empty; if not, we select another particle and repeat the destination selection procedure. Once the particle has been moved, if the new position has any nearest neighbor site belonging to the aggregate, the present position of the walker is added to the aggregate (the cathode or bottom boundary at the initial stage) with probability $s$ (and is able to diffuse over the aggregate surface if that aggregate position has just one nearest neighbor belonging to it; see the following section); otherwise it stays there (and is able to move again) with probability $1-s$. We term $s$ the sticking probability; it is related to the chemical activation energy the cation needs to stick to the aggregate. As particles are added to the aggregate, others are created at the top of the lattice, keeping the mean cation concentration $c$ constant, which in fact simulates an infinitely high system (experimentally this means that the distance between electrodes is much larger than their lateral dimension); consequently, the flux of particles is constant at every stage of the simulation.

As we have already pointed out, the model parameters are related to the physical factors influencing the problem. Indeed, the choice of jump probabilities for the random walkers in the bath provides a recurrence relation which is a discretized version of the continuous equations (1a) and (1c). Therefore, the drift velocity $\mu_{c} \mathbf{E}$ is proportional to the bias $p$. When a finite number of walkers is considered with concentration $c$, we must take into account the excluded volume, so the effective diffusion coefficient and the effective drift velocity in the simulations are proportional to $1-c$ (in a mean field approach) [41]. It is important to note that when $c \rightarrow 0$, i.e., the bath is formed by one particle alone (as in DLA), the aggregate develops tall branches which grow at the expense of short ones due to screening effects. Therefore, in the low-current limit a morphological instability appears that is not always present in ECD experiments. The finite concentration and the hard core interaction among random walkers simulate the cation pressure on the aggregate, so $c$ is an essential ingredient in the understanding of the formation of electrodeposits and to prevent these instabilities (of Laplacian character) from dominating the whole growth process.

One important task is the definition of the simulation time step. In [16], comparison with the experiments in [35] allowed a demonstration that the physical time and the simulation time measured in number of Monte Carlos trials were simply proportional to each other. For this reason, we have stuck to the definition of the time step in [16] as a Monte Carlo trial, i.e., the time needed for a particle to jump, either if the particle does jump or if it does not. Notwithstanding, we have tried other time steps definitions, such as the Monte Carlo step being defined as the mean time for every random walker to jump at least once, but the results are basically the same. Some authors define the time step for the solid-onsolid growth models as the mean time needed to complete an aggregate layer, but, as we will show below, ECD electrodeposits do not grow with constant velocity, and therefore the mean interface height does not grow linearly with time. We thus believe that, in the ECD context, this time unit would be rather artificial and hence we have not used it. In fact, as we will show below, the work reported in this paper provides further evidence in favor of our choice (see the discussion of the experiments in [24] in Sec. III B below).

\section{NUMERICAL RESULTS}

\section{A. Morphologies}

We begin the summary of our results by discussing the morphologies generated by MBDLA with and without SD and the influence of the different rules for SD on them. In addition, we want to compare our computer generated morphologies to the available experimental data. We take as a reference the comprehensive experimental work of Trigueros et al. [11], who reported a systematic experimental study of different growth regimes at constant applied voltage conditions. Their work gave rise to a diagram of morphologies divided into different regions in which similar morphologies were obtained as a function of the applied voltage and the electrolyte concentration. It is important to realize that, in galvanostatic conditions, there is no linear correspondence between voltage and electric current of ions, and therefore, comparison between our morphologies and those reported by these authors can only be qualitative. No similar taxonomic work has been performed for constant current conditions. Although the diagram in [11] is quite complex, it encloses a full variety of morphologies under the label compact. Some authors $[19,21]$ have studied electrodeposit systems within this regime, and hereafter we will also refer to them. Finally, a recent work by Schilardi et al. [24] provides exhaustive information on the asymptotic ECD regimes, which have not been considered anywhere else; hence, their research will also be compared to ours throughout the paper.

As we have already mentioned, from the model perspective we can compare the bias $p$ with the electric current density $[16,42]$, and $c$ with the electrolyte concentration, even though the two latter magnitudes are not exactly coincident, i.e., an electrolyte concentration equal to $0.1 M$ does not mean $c=0.1$. We will see below that the results are not very sensitive to the specific value of $c$ insofar as it is not very small, and thus the difference between actual and model concentrations is not very relevant. The sticking probability $s$ and the diffusion parameters, namely, $l, \lambda$, and $r$ (or equivalently $\tau_{d}$ ), cannot be directly tuned in an experiment, although it is reasonable to expect that changes in the experimental conditions will correspondingly modify these parameters. How much they are modified is something we will learn through our computer simulations.

\section{Bias vs sticking probability without $S D$}

Figure 1 shows a diagram of morphologies obtained with $0 \leqslant p \leqslant 5$ and $0.01 \leqslant s \leqslant 0.5$ without $S D$, with a particle concentration $c=0.05$. We have included these results for two reasons: First, there has been no previous report to our knowledge on MBDLA morphologies, except for a brief discussion in [16]; and, second, we need to discuss them in order to understand later what is the effect of SD on MBDLA morphologies. It is clear from Fig. 1 that increasing the bias or decreasing the sticking probability yields denser aggregates, the ones obtained for $p=0$ and $s=1$ (bottom right) being multiparticle DLA-like as expected (compare to $[31,32])$. This phenomenon is related to the stabilizing effect 


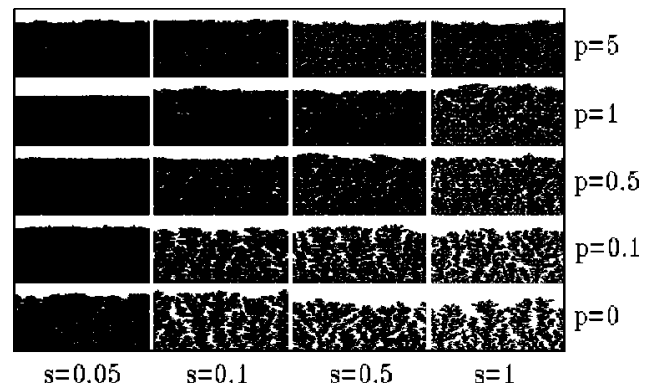

FIG. 1. Morphologies obtained with MBDLA without surface diffusion for $256 \times 400$ systems with a cation concentration $c$ $=0.05$. Other parameters are as indicated in the figure.

of the parameters $p$ and $s$, which can be theoretically demonstrated [43]. Indeed, the higher the value of $p$, the larger the flux of particles reaching the interface in the direction perpendicular to the cathode. This reduces the probability for a cation to stick laterally to a branch and the screening effects due to the Laplacian field. On the other hand, the electric field combined with the reduction of the sticking probability tends to fill the interface valleys. This first result, namely, the fact that increasing the electric current leads to denser aggregates, is similar to the results reported by Trigueros et al. [11], who observed densification of the aggregates with increasing applied voltage. In particular, we can qualitatively compare the morphological changes obtained by varying the bias $p$ for a fixed $s=0.5$ in Fig. 1 with those provided by experimental voltage variations (see Fig. 2 in [11]). We conclude that high voltages (or, in general, high-density currents) yield denser aggregates. So the bias $p$ is an essential ingredient in any realistic ECD model.

As a second step in our study, we have monitored other relevant quantities which in turn can be experimentally measured, in order to obtain additional information aside from qualitative morphological comparisons. Figure 2 shows the local concentration of particles in the bath, still without SD, at equal time intervals. We have plotted the concentration profiles in the stationary regime, i.e., after the instability occurs (see below). Thus, the mean number of attached particles per unit time (or, equivalently, the mean interface velocity) is constant. Léger et al. [8,9] have reported experimental evidence consistent with this stationary behavior (see, e.g., Fig. 5 in [9]). We thus see that MBDLA agrees well with their findings, i.e., the stationary concentration of particles in the bulk approximately obeys the equation $[9,43]$

$$
C(z)=c_{a}+\left(c_{0}-c_{a}\right) e^{-\left(z-z_{0}\right) u / D_{c}},
$$

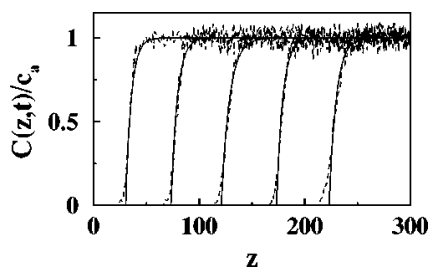

FIG. 2. Concentration profiles for a $512 \times 300$ system with parameters $p=1, s=1$, and $c=0.1$ without surface diffusion. Dashed lines represent the simulation data and solid lines the best fit of those data to Eq. (4). The height $z$ is given in lattice spacings.

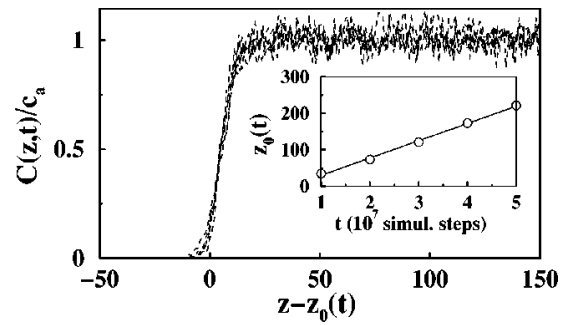

FIG. 3. Collapsed concentration profiles using the values of $z_{0}$ obtained from Eq. (4). Inset: the mean concentration front position $z_{0}$ vs time.

where $z$ is the vertical coordinate, $z_{0}$ is the interface mean position, $c_{a}$ is the concentration at the anode, $c_{0}$ is the concentration at the interface, $u=\mu_{c} \mathbf{E}$, and $D_{c}$ is the bulk diffusion coefficient. As shown in Fig. 2, this function provides a good fit of our data. In Fig. 3, we plot a fit of Eq. (4) (dashed line) to the simulation results, showing a good collapse of the bulk particle density outlines for different times. The small deviations close to the aggregate are due to the interface roughness. The ratio $D / u$ is called diffusion length. In our fits, this length turns out to be about 15 lattice spacings, that is, about two or three times the lateral width of the branches for the chosen parameters. This result provides another check of the physical validity of our model, as we can compare the length obtained from the fit with that taken from Ref. [9]. In this paper, the diffusion length is of order 0.5 $\mathrm{mm}$, about twice the typical branch lateral width (of order 1 $\mathrm{mm}$ ), so we may conclude that the diffusion length obtained from our model is physically consistent.

The inset in Fig. 3 shows the mean concentration front position $z_{0}$ vs time, demonstrating that, in the stationary state, MBDLA leads to a constant velocity of the advancing front, as in the experiments.

\section{Physical relevance of $S D$}

The previous subsection shows that MBDLA without SD successfully reproduces some ECD experiments, in particular, under galvanostatic conditions with not very small electric current density. However, within the MBDLA model it is impossible to understand the unexpected compactification of aggregates in low-voltage experiments $[11,19,21]$ or the columnarlike growth found in other situations. Unfortunately, MBDLA aggregates are always ramified at low bias. In [16], a phenomenological explanation of compactification was proposed by noticing that the reduction of $s$ leads to more compact aggregates. Therefore, it was proposed there that $p$ and $s$ should be related by a monotonic function, the simplest case being that of a linear relationship. With this procedure, reducing the bias leads to a corresponding decrease in the sticking probability, and hence to compact aggregates at low bias. However, this is an ad hoc assumption that cannot be experimentally tested, whereas its theoretical justification is not very clear. Besides that, this approximation does not reproduce other morphologies, such as those reported by López-Salvans et al. [18] and Kahanda et al. [21]. In view of this, it became increasingly clear that there was some crucial ingredient missing in MBDLA, and the most obvious candidate was, of course, SD. 
At this point, it is instructive to consider carefully the work by Kahanda et al. [21]. According to their results, as the absolute value of the overpotential decreases, the aggregate becomes denser, and it is formed by several columns that are thicker at the top than at the bottom. We interpret this as a hint of the relevance of SD: If, when a particle arrives at the top of a column, it diffuses along the aggregate interface, and if the diffusion length is shorter than the column perimeter, the particle will not reach the base of the pillar or another column, with the result of a characteristic inverted triangle structure. The onset of similar triangle structures has also been reported by Pastor and Rubio [19]. We thus came to the conclusion that it was necessary to include SD in MBDLA in order to shed further light on the nontrivial coupling of the different transport mechanisms.

\section{Implementation of $S D$ in MBDLA}

We have implemented SD in MBDLA in three different ways, all of them starting when a particle in the bulk (the electrolytic solution) sticks to the aggregate but has just one neighbor. We first tried two simple irreversible rules (other similar rules yield equivalent results, so we do not include them here for brevity), named rules $A$ and $B$, and a reversible one, named rule $C$.

Rule A. The newly incorporated particle always jumps in the same direction, either left or right, parallel to the cathode, until it reaches a site with at least two neighbors or completes $l$ jumps. This rule is similar, but not identical, to the one studied in [44] for ballistic deposition with surface diffusion.

Rule $B$. In this second rule, we allow the particle to perform a random walk over the aggregate surface until it increases its coordination number, with a constant probability $\lambda$ to be permanently stuck to its current position (this is the so called mortal random walker [45]).

The last rule is characterized by Arrhenius-like jump probabilities and, what is more important, by simultaneous bulk diffusion and SD.

Rule $C$. This rule allows several particles to diffuse simultaneously. When a particle arrives at a coordination 1 site, it sticks and jumps to one of its two nearest neighbors on the aggregate with probability $p_{n}=\exp \left[-E_{0}+(n-1) E_{a}\right]$, where $E_{0, a}$ are adimensional activation energies, and $n$ is the coordination number of the target position. If the particle's new position has two or three neighbors, it attaches to the aggregate irreversibly. Otherwise, we label the particle as a SD particle, and we allow it to take further steps. Thus, we have two kinds of diffusing particles: particles in the bulk, distributed homogeneously with concentration $c$, and particles that diffuse over the aggregate surface. With probability $r$ we choose a bulk particle that evolves with its characteristic rules, and with probability $1-r$ a particle on the surface that jumps to one of its nearest neighbors as we have just described for the first jump. This rule is close in spirit to the collective diffusion rules employed in studies of kinetic roughening in molecular beam epitaxy (MBE) [46], and in particular to MBE models beyond the solid-on-solid approximation $[47,48]$.

The main difference between rules $A$ and $B$ with respect to rule $C$ is that the latter introduces a characteristic time scale $\tau_{d}=r^{-1}$, while in the other cases diffusion is instanta-

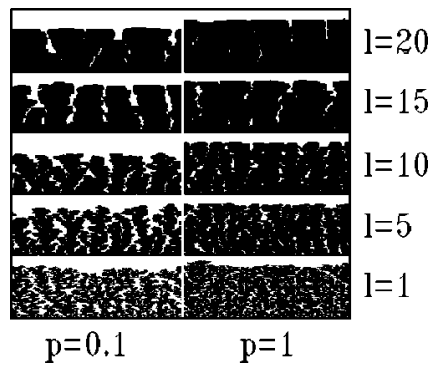

FIG. 4. Morphologies obtained with MBDLA with surface diffusion rule $A$ and parameters $s=1$ and $c=0.1$. The size of the system is $256 \times 400$ pixels.

neous; thus, the diffusing particle is not affected by the overall particle dynamics. As we will show below, rule $C$ is the only one that actually reproduces the influence of SD on the aggregate scaling and morphology. In this respect, it is important to point out that we have found that Arrhenius-like probabilities alone are not enough to model SD: variants of rule $C$ with those probabilities and without the characteristic time, i.e., SD kept instantaneous, lead once again to results similar to those of rule $B$. All the results presented were obtained with $E_{0}=3$ and $E_{a}=1$. We have chosen these values to have jump probabilities smaller than 1 , but other sets of parameters yield similar results, which we omit for brevity. Finally, another interesting point is that the probabilities in rule $C$ allow one to trivially introduce temperature in the model by simply identifying $E_{0, a} \rightarrow E_{0, a}^{\prime} / k_{B} T$.

Rule $A$, by definition, introduces a diffusion length $l$, but if $l \gg 1$ the particle jumps essentially always lead to an increment of its coordination, as may be seen in Fig. 4, where some morphologies are shown for different values of $l$. The inverted triangle structure typical of the experiments by Kahanda et al. [21] is reproduced with this simple rule. Nevertheless, the tops of the pillars are unrealistically flat; another problem is that decreasing $p$ does not yet lead to a compact aggregate regime. Rule $A$ is therefore not appropriate. In the case of rule $B$, the diffusion length is introduced indirectly by means of the attachment probability $\lambda$ (see the Appendix for details). The mean diffusion length can be shown to be given by $l_{D}=1 /\left(2 \lambda^{1 / 2}\right)$. Morphologies obtained with this rule are plotted in Fig. 5. Once again, and in spite of the fact that rule $B$ allows the particles to diffuse randomly over the aggregate, the columns developed during the growth turn out completely flat at the top, and the option of rule $B$ was excluded as well.

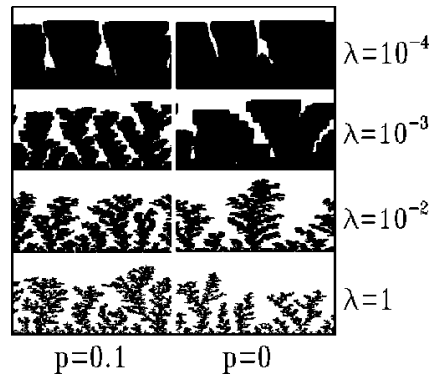

FIG. 5. Morphologies obtained with MBDLA with surface diffusion rule $B$ and parameters $s=1$ and $c=0.1$. The size of the system is $256 \times 400$ pixels. 


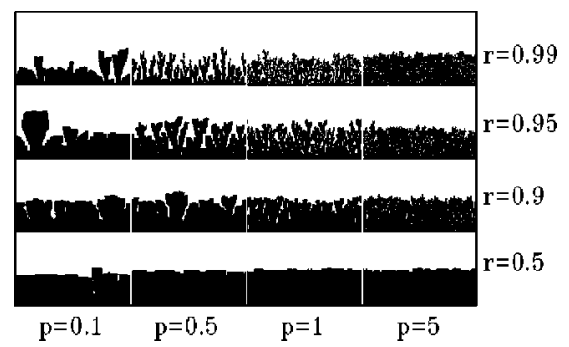

FIG. 6. Morphologies obtained with MBDLA with surface diffusion rule $C$ and parameters $s=1$ and $c=0.1$. The size of the system is $256 \times 400$ pixels.

These pitfalls (and similar ones found by using Arrheniuslike jump probabilities, which we skip for brevity) led us to the conclusion that instantaneous SD (or limited mobility rules, in the terminology of [46]) is a too drastic approximation for ECD. Taking into account that the main distinctive feature of MBDLA is its nonlocal character, interactions of particles diffusing along the surface with newly deposited particles are expected to be relevant. Guided by these ideas, we propose rule $C$, which incorporates this coupling by introducing time scales for both bulk diffusion and SD. A sample of the aggregates generated by MBDLA with rule $C$ is shown in Fig. 6. The difference from the other two rules is immediately apparent from the plot: This more realistic rule does induce the creation of pillars as we pointed out above, this time similar to those reported by Kahanda et al. [21] and Pastor and Rubio [19], which are rough at the top. Moreover, the compactification of the aggregates at low currents appears naturally, as can be noted by following the sequence of aggregates appearing on the same row (same value of $r$ ): decreasing the current leads initially to less dense aggregates, until further reduction of the current gives rise to more compact aggregates. Remarkably, there is no need to change the sticking probability by hand as in MBDLA without SD or with rules $A$ and $B$. This allows us to eliminate one model parameter, the sticking probability, which we take to be $s$ $=1$ from now on.

So far, we have seen that, while simple SD rules provide good results in some solid-on-solid simulation models, the complex dynamics of Laplacian systems does not allow the particles to instantaneously diffuse; rather, we must allow several particles to interact before they become permanently stuck to the aggregate. Roughly speaking, the flux of particles arriving at the aggregate defines a characteristic time $\tau_{p}$ (typically inversely proportional to the flux, i.e., to $p$ ). Once the particles have arrived at the aggregate, they diffuse until they reach a site with coordination larger than 1, or, equivalently, until the particle meets another diffusing particle, thus forming a dimer on the interface that cannot move anymore. A large flux of particles arriving at the interface (large $p$ ) will increase the probability of formation of those dimers, and the particles can hardly diffuse. The situation is not so simple when $p$ is small. On one hand, the deposition mean time $\tau_{p}$ is large, but on the other hand, the particles hardly experience the applied electric current, so the probability of attachment to a column wall before getting to the bottom of the aggregate increases. Thus the Laplacian instability is amplified, leading to a compact structure formed of columns and grooves. This kind of instability has been ob-

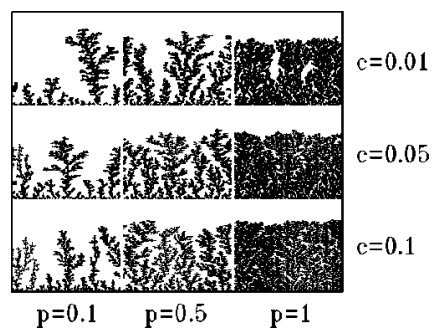

FIG. 7. Morphologies obtained with MBDLA without surface diffusion for parameters $s=1$ and $r=1$. Other parameters are as indicated.

served in low-current galvanostatic experiments [19]. The aggregate is therefore denser but if the diffusion time is not long enough the interface is unstable. It is remarkable that this simple picture in terms of time scales allows to understand the relevance of SD in ECD experiments.

A final important remark we would like to make is that, when the diffusion probability $r$ is about 0.99 , we have observed some evidence of what could be a morphological transition (and the subsequent change in the branches) similar to those reported by López-Salvans et al. [17]. However, as we want to concentrate in this paper on MBDLA with SD as a generic model for all regimes of ECD experiments, we postpone a more careful study of this possibility to future work, where we will pursue the appearance of this phenomenon for different model parameters (such as $p$ or $r$ ).

\section{Electrolyte concentration}

To conclude the analysis of MBDLA parameters, we show the effect of the electrolyte concentration $c$. Figure 7 exhibits the morphological changes in patterns with different $c$ values ranging from 0.01 to 0.1 for different bias $p$ without $\mathrm{SD}$. Note that when $c \rightarrow 0$ the low-current limit is exactly the DLA growth model [25]. Therefore, we should keep a finite value of $c$ in order to diminish the unavoidable DLA characteristic instability. The results contained in the figure allow us to conclude that, insofar as $c$ is not very small, the morphologies obtained with MBDLA do not depend strongly on the concentration, and therefore the fact that there is no direct correspondence between physical and simulated concentrations is not a drawback of the model.

\section{B. Dynamic scaling}

The previous subsection shows that inspection of the morphologies is a valuable method to check the validity and relevance of the model rules. Indeed, the unrealistically flat aggregates obtained with diffusion rules $A$ and $B$ disqualify them and motivate the investigation of the more realistic, noninstantaneous rule $C$ for SD. However, in order to exploit the main virtues of MBDLA with SD and to compare with other relevant models and experiments, we must take some quantitative criteria, for example, the analysis of the interface surface roughening. To this end, let us define some functions related to the height of the aggregate at spatial position $x$ at time $t$, given by the scalar field $h(x, t)$. We will also review their basic features before discussing MBDLA properties.

The global width (or roughness) $W(L, t)$ is nothing but the rms fluctuation of the height variable $h(x, t)$ around its 
mean value $\bar{h}_{L}(t)=(1 / L) \Sigma_{x} h(x, t)$ :

$$
W^{2}(L, t)=\frac{1}{L}\left\langle\sum_{x}\left[h(x, t)-\bar{h}_{L}(t)\right]^{2}\right\rangle,
$$

where angular brackets stand for the ensemble average. Generally speaking, in many growth models, starting from $h(x, 0)=0$ the width satisfies the dynamic scaling hypothesis of Family and Vicsek [49]:

$$
W(l, t) \sim\left\{\begin{array}{lll}
t^{\beta} & \text { if } & t \ll L^{z} \\
L^{\alpha} & \text { if } & t \gg L^{z}
\end{array}\right.
$$

The roughness exponent $\alpha$, the dynamic exponent $z$, and their ratio (growth exponent) $\beta=\alpha / z$ identify the universality class the model belongs to.

In the study of kinetic roughening the height-height correlation function is frequently used [2]:

$$
C^{2}(l, t)=\frac{1}{L}\left\langle\sum_{x}[h(x+l, t)-h(x, t)]^{2}\right\rangle,
$$

where $[50,51]$

$$
C(l, t) \sim \begin{cases}t^{\beta} & \text { if } t \ll l^{z}, \\ t^{\left(\alpha-\alpha_{l o c}\right) / z} l^{\alpha l o c} & \text { if } t \gg l^{z},\end{cases}
$$

and $\alpha_{l o c}$ is the so called local roughness exponent. Another important function related to the height variable $h$ is the power spectrum,

$$
S(k, t)=\langle\hat{h}(k, t) \hat{h}(-k, t)\rangle
$$

where $\hat{h}(k, t)=L^{-1 / 2} \sum_{x}\left[h(x, t)-\bar{h}_{L}(t)\right] \exp (i k x) . S(k, t)$ displays a behavior consistent with the scaling form [52]

$$
S(k, t)=k^{-(2 \alpha+1)} s\left(k t^{1 / z}\right),
$$

where

$$
s(u)=\left\{\begin{array}{lll}
u^{2 \theta} & \text { if } & u \gg 1, \\
u^{2 \alpha+1} & \text { if } & u \ll 1 .
\end{array}\right.
$$

The exponent $\theta$ takes different values depending on the type of scaling exhibited by the model. For instance, for the so called intrinsic anomalous scaling [52] we have $\theta=\alpha$ $-\alpha_{l o c}$, whereas $\theta \equiv 0$ for Family-Vicsek scaling (including super-roughening, i.e., $\alpha \geqslant 1$ ). Note that this implies $\alpha$ $=\alpha_{l o c}$.

To apply these ideas to MBDLA characterization, a few remarks are in order. Although, in some cases, MBDLA develops ramified aggregates leading to multivalued interfaces, i.e., interfaces with overhangs, it has been demonstrated [53] that the interface of the active zone in DLA simulations (the aggregate sites with larger probability of arrival) corresponds to that constructed by taking the topmost site $h(x, t)$ at every horizontal position $x$. This construction does not ensure that the measured exponents are free of interpretation [54], but the exponents are consistent with theoretical and experimental data [55]. The reduction of the sticking probability $s$ yields denser aggregates, and overhangs do not appear at any stage of the simulation for low $s$ values. Besides that, if SD is present the aggregates are also more compact. In all these cases the function $h(x, t)$ is identical to the aggregate outline and consequently the results do not have any interpretation problem.

The main scaling features of MBDLA without SD were already reported in [23]. Therefore, here we will briefly summarize them to facilitate comparison with results including $\mathrm{SD}$, and refer the reader to [23] for the details. Without SD, MBDLA displays three temporal regimes: At early times the global width $W(L, t)$ features $\beta=0.5$, this value being simply due to shot noise. This stage corresponds to times at which the lateral correlation length is of the order of the lattice spacing. After this noisy transient, short and large length scales are governed by different dynamics because the bulk Laplacian field produces nonlocal effects (screening or shadowing among branches). Consequently, the local and the global roughness exponents $\alpha_{l o c}$ and $\alpha$ are different and the interface is not self-affine. The growth exponent $\beta$ is larger than that of noise $(\beta>1 / 2)$ because some isolated branches begin to grow independently from each other, which can be understood as a signature of Laplacian instability. As a consequence, the interface width grows rapidly as compared with the noise fluctuations. At later times, branches spread by lateral growth and impinge upon each other. Eventually, the system reaches an asymptotic regime characterized by the Kardar-Parisi-Zhang (KPZ) universality class [56] exponents $(\alpha=1 / 2, \beta=1 / 3, z=3 / 2)$. The KPZ equation is the paradigmatic growth model without $\mathrm{SD}$, and it is given by the stochastic partial differential equation [56]

$$
\frac{\partial h}{\partial t}=\nu \nabla^{2} h+\frac{\lambda_{0}}{2}(\nabla h)^{2}+\eta(x, t)
$$

where $\nu$ and $\lambda_{0}$ are constants and $\eta(x, t)$ is a Gaussian white noise with

$$
\langle\eta(x, t)\rangle=0,
$$

$$
\left\langle\eta(x, t) \eta\left(x^{\prime}, t^{\prime}\right)\right\rangle=2 D \delta\left(x-x^{\prime}\right) \delta\left(t-t^{\prime}\right) .
$$

As mentioned above, the definition of the interface function $h(x, t)$ neglecting overhangs might cast some doubts [54] on the validity of the exponents reported in [23]. To confirm our results, we have measured the excess velocity produced by tilting the initial substrate and imposing helicoidal boundary conditions [2]. The inset in Fig. 8 shows that this mean velocity is well fitted by a parabola, as expected for KPZ behavior. It is important to stress that identical results are obtained using the jump rules in [23].

Interestingly, Schilardi et al. [24] report experiments with large currents (equivalent to the large values of the bias $p$ ) in excellent agreement with our model. They observe the same three time regimes: An initial transient with a behavior that could not be measured due to the resolution of the experimental device, a second transient with $\beta>1$ characterized by the growth of isolated branches, and a third asymptotic regime at which the interface is characterized by KPZ exponents. A plot of the mean interface velocity vs time is also given, showing a crossover from the unstable regime to the stable one in accordance with MBDLA predictions, as can be 


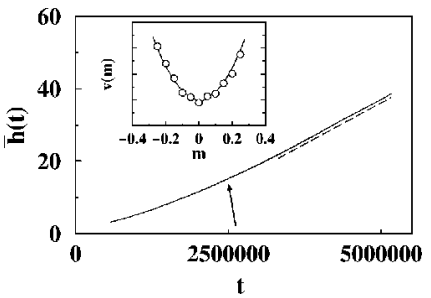

FIG. 8. Interface mean height vs time with parameters $p=4, s$ $=1$, and $c=0.05$ without surface diffusion. The arrow shows the end of the unstable regime. Inset: mean excess velocity, in arbitrary units, for the same parameters for different boundary tilts $m$. Circles stand for simulation and the solid line is the best fit to a parabola. The dashed line represents the expected linear growth of KPZ type.

seen in Fig. 8. The global width crosses over from the instability $(\beta>0.5)$ to $W(L, t) \sim t^{1 / 3}$ at the time pointed out by the arrow. Note that MBDLA cannot yield $\beta$ larger than 1 , because of its discrete growth rules. This would mean that the interface width grows faster than the interface mean height. Finally, the evolution of the morphology during the experiment is also the same in MBDLA and in the experiment, as seen by comparing Fig. 9, taken from [24], and Fig. 10, obtained in our simulations.

We now consider the scaling behavior of MBDLA + SD for the different diffusion rules. As we pointed out in the preceding section, large values of the diffusion length $l$ (rule A) generate flat aggregates. This means that $\beta \rightarrow 0$ as $l \rightarrow \infty$ at early times. Figure 11 shows the lack of universality in the

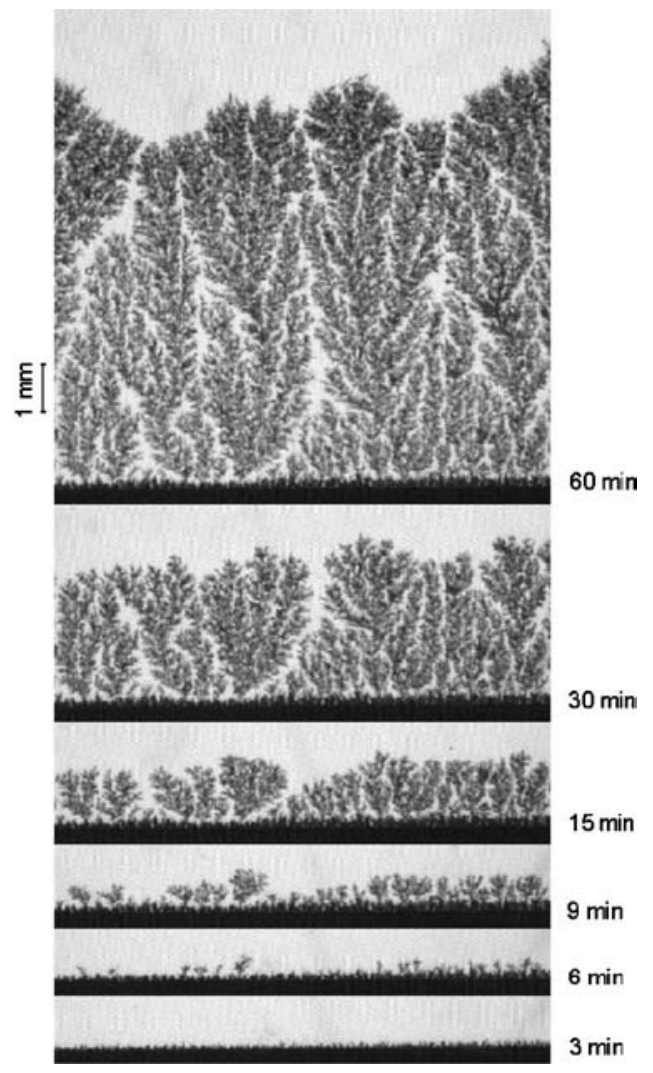

FIG. 9. In situ lateral micrographs showing the interface evolution from $t=0$ to $t=60 \mathrm{~min}$ for $\mathrm{Ag} \mathrm{ECD}$ at $j=1 \mathrm{~mA} \mathrm{~cm}^{-2}$ in ( 5 $\left.\times 10^{-3}\right) \mathrm{MAg}_{2} \mathrm{SO}_{4}+10^{-2} \mathrm{MH}_{2} \mathrm{SO}_{4}+0.5 M \mathrm{Na}_{2} \mathrm{SO}_{4}$. Taken from [24] with kind permission from the authors.
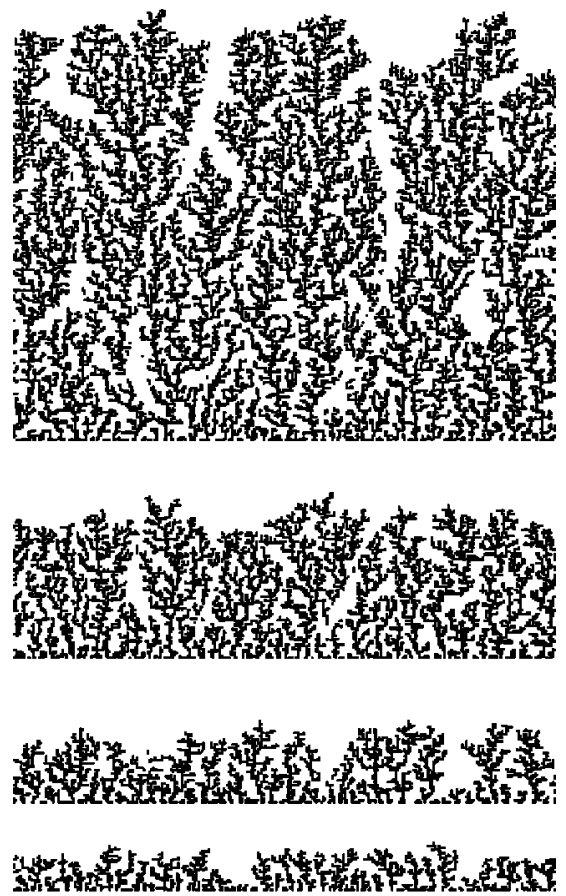

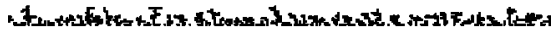

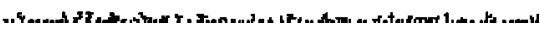

FIG. 10. Sequence of snapshots of the evolution of an aggregate grown with MBDLA without SD. Parameters are $p=0.75, s=1$, $r=1$ (i.e., no surface diffusion). Times (in our units, see text) are (top to bottom) $35 \times 10^{6}, 17.5 \times 10^{6}, 8.75 \times 10^{6}, 5.25 \times 10^{6}, 3.5$ $\times 10^{6}$, and $1.75 \times 10^{6}$.

growth exponent $\beta$ : It can be seen in this plot that $\beta$ decreases with $l$ as we expected. The same happens with rule $B$ : As in the case of rule $A$, the growth exponent $\beta$ depends strongly on the attachment probability, $\lambda$ (rule $B$ ). As depicted in Fig. 11, the dependence is similar to that of model A since the diffusion length is proportional to $\lambda^{-1 / 2}$.

The scaling behavior in MBDLA with SD given by rule $C$ is more complicated. We can recognize three different kinds of behavior, which we summarize as follows.

$0.05 \leqslant r \leqslant 0.25$. The characteristic diffusion time is long, and particles diffuse rather fast along the surface (let us recall that they are picked with probability $1-r$ at every Monte Carlo trial) without much interaction with particles arriving from the bulk, thus yielding compact aggregates, except if $p \leqq 0.05$, because then the Laplacian field creates pillars and grooves. After a short transient the global width grows slowly and, independently of the applied current, the roughness exponents are compatible with those of the

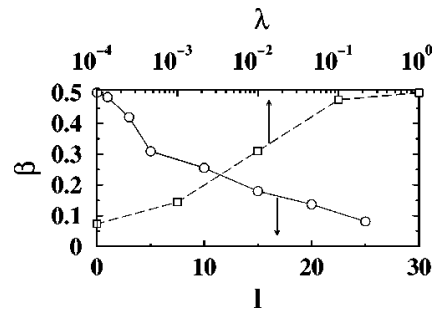

FIG. 11. Dependence of the growth exponent $\beta$ on $(\bigcirc)$ diffusion length $l$ (rule $A$ ) and $(\square)$ attachment probability $\lambda$ (rule $B$ ). 


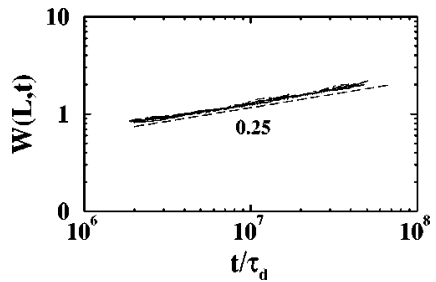

FIG. 12. Global width vs $t / \tau_{d}$ for $r=0.05,0.1,0.15,0.2$, and 0.25 , with $p=0.5, s=1$, and $c=0.05$. The dashed line is a guide to the eye, with slope 0.25 .

Edwards-Wilkinson universality class, whose defining equation is [57]

$$
\frac{\partial h}{\partial t}=\nu \nabla^{2} h+\eta(x, t)
$$

Figure 12 shows the global width collapse obtained by rescaling the simulation time. The plot not only shows the Edwards-Wilkinson growth exponent, but also the $r$ independence of the results over a wide range of simulation parameters. Note that the collapsing time step is the one defined for MBDLA without SD divided by the characteristic diffusion time $\tau_{d}=r^{-1}$. Figure 13 shows the collapsed power spectrum using $\alpha=1 / 2$ and $z=2$ (and consequently $\beta=1 / 4$ ) consistent with Eq. (10) with $\theta=0$ for Edwards-Wilkinson exponents. It is important to note that this kind of dynamic scaling has been observed in two-dimensional ECD experiments [58]. Finally, we have to mention that the restriction $r>0.05$ is due only to the extremely long computational times needed to study the model for such small values of $r$.

$0.3 \leqslant r \leqslant 0.7$. For large $p$, the interface is compact and grows with constant velocity. The scaling is similar to that of the preceding case. When $p \rightarrow 0$, initially the interface is rough and the growth exponent $\beta$ is in the range $0.35-0.40$ (see Fig. 14). Some experiments have reported similar interfaces at early stages of growth [20]: Specifically, they obtained exponents consistent with the linear MBE growth model universality class $(\alpha=3 / 2, \beta=3 / 8=0.375$, and $z$ $=4$ ) that is, their interfaces could be described by the equation [59]

$$
\frac{\partial h}{\partial t}=-K \nabla^{4} h+\eta(x, t) .
$$

Note that for this model $\alpha>1$, so the interfaces generated with Eq. (14) are super-rough. In our case this short regime

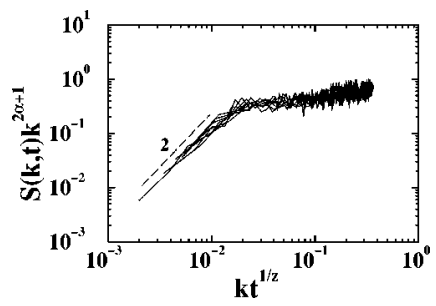

FIG. 13. Collapsed power spectrum with $p=2, s=1, r=0.1$, and $c=0.05$ using the Edwards-Wilkinson universality class exponents at six equally spaced times from $8 \times 10^{6}$ to $3 \times 10^{7}$. Dashed line has slope $2 \alpha+1=2$.

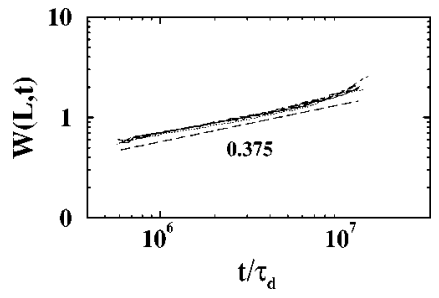

FIG. 14. Global width vs $t / \tau_{d}$ for $r=0.35,0.40,0.45,0.55$, and 0.60 , with $p=0.5, s=1$, and $c=0.1$. Dashed line is a guide to the eye.

ceases when the mean interface height $\bar{h}(t)$ is about 8 to 10 monolayers and the global width $W(L, t)$ is about 1 . This is compatible with the referred experiments referred to except that we do not observe the super-rough power spectrum. Actually, in our case the tail of $S(k)$ presents a time shift at large wave vectors (Fig. 15), which is incompatible [60] with the behavior obtained for Eq. (14). However, the basic phenomena, such as the value of the effective $\beta$ and the onset of the instability, are in good agreement with the experiments. After this transient, the aggregates are still compact and develop some grooves (see Fig. 6). When these grooves appear, the growth exponent $\beta$ rises dramatically due to the large slopes produced between grooves. Figure 16 summarizes all this by showing the variation of $\beta$ with time.

$0.85 \leqslant r$. Finally, when the diffusion time is short, three completely different situations are found as a function of the current $p$. For very large $p$, cations become ballistically driven to the aggregate and the unstable transient tends to dissappear (in fact, the $p \rightarrow \infty$ limit is the ballistic depostion discrete model, which is well known to belong to the KPZ universality class [1,2]). When $p \geqslant 1$ the aggregate grows as MBDLA without SD with similar parameters, except that in this case the aggregate mean density rises. That is, we successively detect a noisy initial transient, the instability associated with the growing branches, and the KPZ asymptotic limit due to the lateral growth of the branches. The interfaces within the unstable regime (an example of which is shown in Fig. 17) are not self-affine but present intrinsic anomalous scaling [51,52]. Figure 18 shows the power spectrum for $r$ $=0.85, p=4$, and $s=1$. Figure 19 shows the collapse of the power spectrum and Fig. 20 the collapse of the height-height correlation function $C(l, t)$, achieved in both cases for $\alpha$ $=1.78, \alpha_{l o c}=0.49, z=2.51$, and $\beta=0.71$.

For intermediate $p$ values (between 0.25 and 1 , for almost every $r$ ), the aggregate is formed by several compact thin branches which grow vertically and parallel to one another.

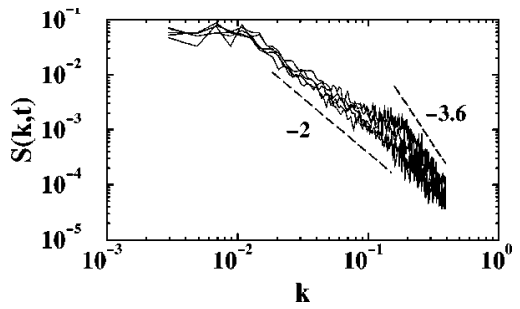

FIG. 15. Power spectrum of an interface with $r=0.5, p=0.5$, $s=1$, and $c=0.1$ at times $10^{7}, 2 \times 10^{7}, 3 \times 10^{7}, 4 \times 10^{7}$, and $5 \times 10^{7}$. Power spectra are anomalous at short scales. Dashed lines are guides to the eye. 


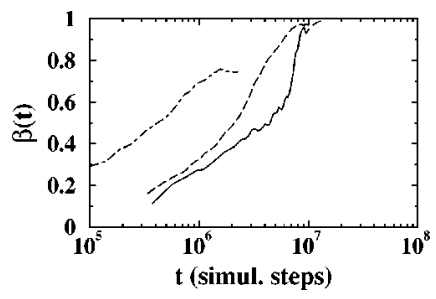

FIG. 16. Evolution of growth exponent $\beta$ with time for compact aggregates with grooves. Solid line: $p=0.1$ and $r=0.45$; dashed line: $p=0.1$ and $r=0.5$; and dot-dashed line: $p=0.1$ and $r=0.7$.

In this case, the notions of a rough surface or dynamic scaling are meaningless. Finally, for small $p$ some compact branches grow at the expenses of the others, so typically one or two branches grow more than the others. As in the case of parallel branches, it is meaningless to talk about interface roughening.

\section{DISCUSSION AND CONCLUSIONS}

Our first conclusion is that MBDLA is a simple computational model that incorporates in a natural way some of the basic mechanisms involved in ECD experiments. The original model $[16,23]$ was already known to be in good agreement with some experiments [35]. In this paper, we have provided much more evidence showing that MBDLA explains some of the morphological changes due mainly to the applied electric current and, what is more important, it predicts the recently observed KPZ scaling behavior in the highcurrent limit (for which SD is not too relevant) [24] and observed also at low currents [21]. Before this regime is reached, there is an unstable transient within which MBDLA interfaces present intrinsic anomalous scaling. We believe this type of scaling occurs because SD is not able to communicate to different portions of the interface fast enough, so that they grow independently from one another. This is analogous to the anomalous scaling occurring in the nonlinear surface diffusion equation studied in [61]. In our case, the different portions feature a value of the roughness exponent $\alpha_{l o c} \approx 0.5$, similarly to the interface subject to columnar disorder studied in [51,52].

Secondly, the main point of our paper is that, as we have seen, MBDLA without SD cannot explain low-current experiments in which the characteristic dense branching aggregates of high-current experiments are replaced by compact and columnlike aggregates. Our working hypothesis was that the latter kind of pattern is due to the competition between the Laplacian field of the cations in dissolution and the SD

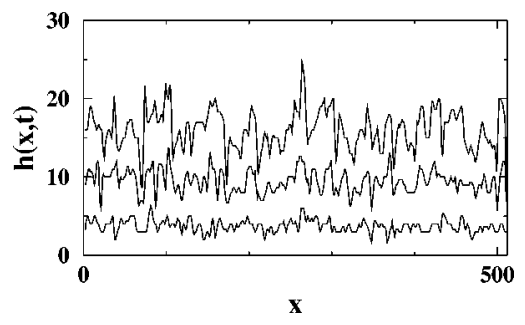

FIG. 17. Dynamic evolution of the height $h(x, t)$ with $p=4, r$ $=0.85, s=1$, and $c=0.1$. Snapshots are taken at times 1.2 $\times 10^{7}, 2.1 \times 10^{7}$, and $3 \times 10^{7}$.

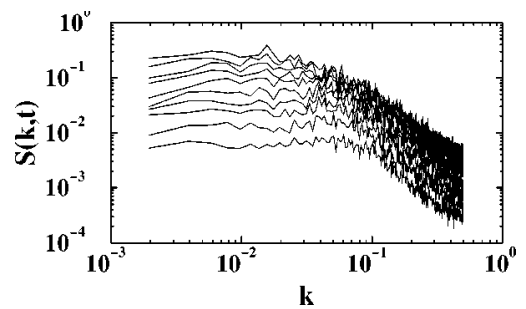

FIG. 18. Intrinsic anomalous power spectrum with $p=4, r$ $=0.85, s=1$, and $c=0.1$. Lines correspond to interfaces at times $3 \times 10^{6}, 6 \times 10^{6}, 9 \times 10^{6}, 1.2 \times 10^{7}, 1.5 \times 10^{7}, 1.8 \times 10^{7}, 2.1 \times 10^{7}$, $2.4 \times 10^{7}, 2.7 \times 10^{7}$, and $3 \times 10^{7}$.

current on the interface. Thus, our ECD model, which we wanted to improve so as to explain, at least qualitatively, the complete ECD phenomenology, should incorporate a new rule for the diffusion of the adatoms attached to the aggregate. Hence, we have tried out some SD rules similar to those often used in growth models for molecular beam epitaxy [46]. We have verified that instantaneous diffusion rules, namely, rules that "freeze'" the bulk particles while the most recently attached particle finds its way through the surface, do not lead to correct results in the low-current limit, and produce very unrealistic, flat-topped morphologies. We have thus been forced to conclude that the nonlocal character of MBDLA demands a diffusion rule that couples the overall cation dynamics, this is the rule we have named $C$. It introduces a characteristic diffusion time $\tau_{d}=r^{-1}$ which competes with the time scale related to the net flux of particles arriving to the interface (which, in fact, is proportional to the applied electric current density). With this SD rule, the morphologies at low, medium, and high currents are compatible with those observed by Trigueros et al. [11] for low, medium, and high applied voltages, respectively. This diffusion time $\tau_{d}$ cannot be controlled from the experimental point of view, but fortunately there are wide ranges of parameters over which the simulated morphologies hardly change, which means that the description of the experiments provided by MBDLA with SD is robust and does not need an uncontrollable parameter to be tuned. We have also compared MBDLA with SD with the experiments reported by Pastor and Rubio [20,19], which characterize the product interfaces by the MBE exponents. MBDLA seems to reproduce the latter behavior for very short times and short length scales, as can be seen in Figs. 14 and 15, but these results are not too significant, as they are not as accurate as we would need to make any strong claim, and could be due to the appearance of a characteristic short length scale. There is a

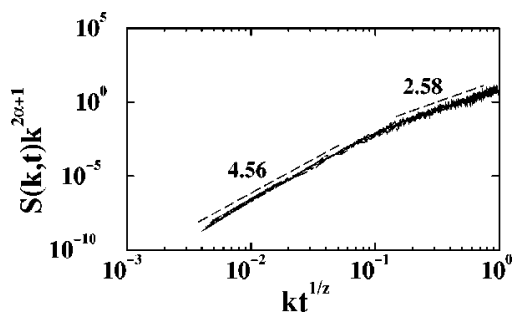

FIG. 19. Collapsed power spectrum for the five later curves in Fig. 18 using $\alpha=1.78, z=2.51, \beta=0.71$, and $\alpha_{\text {loc }}=0.49$. Dashed lines show the slope values expected from Eq. (10) for those exponent values. 


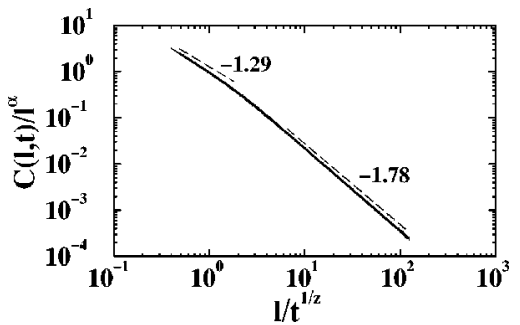

FIG. 20. Collapsed correlation function for the same parameters as in Fig. 19 for five equally spaced simulation times using $\alpha$ $=1.78, z=2.51, \beta=0.71$, and $\alpha_{l o c}=0.49$. Dashed lines show the slope values expected from Eq. (8) for those exponent values.

difficulty in this respect, as MBDLA scaling is intrinsically anomalous, whereas the results in $[20,19]$ support standard, super-rough scaling. With the data presently at hand we have to conclude that MBDLA with SD does not describe all the aspects of the very-low-current regime quantitatively, but the fact that it does describe most of them and, above all, the compactification of the aggregates, makes us confident that MBDLA with SD is a very good general model for ECD.

To conclude, we note that the model presented has the basic ingredients of ECD phenomena, diffusion, electromigration, and surface diffusion, but for this reason, we have to pay a big price in terms of computational time. MBDLA without SD is a very time-consuming model, and the diffusion rules make the analysis and the simulations an exercise in patience. It has certainly been an improvement to find that SD rule $C$ allows us to skip the sticking probability parameter, thus reducing the parameter space, but even then, if averages of relevant quantities over large ensembles are required, a great amount of computational resources will be needed. Of course, this disadvantage can be removed by a careful reprogramming of the algorithm, but that is another line of research. As our goal was to identify the most important factors involved in ECD, we do believe that, despite the computing limitations of the model, MBDLA with SD is a powerful tool to repoduce some unclear features of this kind of growth experiment, and has helped us to understand what are the most relevant transport properties and how they couple in different parameter regions. We hope that this work suggests further experiments to find out whether MBDLA with SD is the complete, general model for ECD or if there are still regions that need separate modeling.

\section{ACKNOWLEDGMENTS}

It is a pleasure to thank Miguel Angel Rodríguez and Francesc Sagués for their help through many discussions on the modeling of ECD and on the subtleties of dynamic scaling during the last few years, as well as for a critical reading of the manuscript. In addition, we are grateful to Javier $\mathrm{Bu}-$ ceta, Juanma Pastor, Javier de la Rubia, and Miguel Angel
Rubio for sharing with us their results and expertise on ECD at very low currents. We would also like to thank Roberto Salvarezza and Leticia Schilardi for their kind permission to reproduce Fig. 9 from [24]. We are indebted to José Cuesta and Ricardo Brito for helpful suggestions about the simulation rules and to the members of GISC for their interest in this work. Work at GISC was supported by DGES (Spain) Grant No. PB96-0119 and CAM Grant No. 07N/0034/98.

\section{APPENDIX: SURFACE DIFFUSION RULE $B$}

In rule $B$, surface diffusion starts when a particle has first arrived at the aggregate and has attached to it (with probability $s$ ). The particle jumps with equal probability to one of its two nearest neighboring sites on the aggregate until it increases its coordination number. The particle has an additional probability $\lambda$ of being permanently attached. This kind of particle is usually termed a mortal random walker [45]. The random walk is performed between two absorbing boundaries, namely, two sites with higher coordination ( 2 or 3 ). One could try to determine a priori the total number $N$ of jumps the particle has to perform in each realization, drawing such a number from the probability for the particle to take $N$ steps on a flat line if it avoids sticking $N-1$ times and "dies" at the $N$ th jump. This probability is easily calculated to be given by

$$
P_{N}(\lambda)=\lambda(1-\lambda)^{N-1} \text {. }
$$

However, the absorbing boundaries disallow this procedure. In any case, we have compared the simulation results by allowing the particle to perform an actual mortal random walk, and to perform a simple random walk of $N$ steps given by Eq. (A1). The two results are hardly different. Thus, we can approximately calculate from Eq. (A1) the mean and variance of the maximum number of jumps, given by

$$
\begin{gathered}
\bar{N}=\frac{1}{\lambda}, \\
\sigma_{N}=\frac{\sqrt{1-\lambda}}{\lambda} .
\end{gathered}
$$

For a flat interface, the particle mean position would be 0 but its variance would be

$$
\sigma_{N}=\bar{N}^{1 / 2} / 2=1 /\left(2 \lambda^{1 / 2}\right)
$$

which provides the characteristic diffusion length $l_{D}$ $=1 /\left(2 \lambda^{1 / 2}\right)$.
[1] P. Meakin, Fractals, Scaling, and Growth far from Equilibrium (Cambridge University Press, Cambridge, 1998).

[2] A.-L. Barabási and H. E. Stanley, Fractal Concepts In Surface Growth (Cambridge University Press, Cambridge, 1995).
[3] M. Matsushita, M. Sano, Y. Hayakawa, H. Honjo, and Y. Sawada, Phys. Rev. Lett. 53, 286 (1984).

[4] D. Grier, E. Ben-Jacob, R. Clarke, and L. M. Sander, Phys. Rev. Lett. 56, 1264 (1986). 
[5] J.-N. Chazalviel, Phys. Rev. A 42, 7355 (1990).

[6] G. Marshall and P. Mocskos, Phys. Rev. E 55, 549 (1997).

[7] V. Fleury, M. Rosso, J.-N. Chazalviel, and B. Sapoval, Phys. Rev. A 44, 6693 (1991), and references therein.

[8] C. Léger, J. Elezgaray, and F. Argoul, Phys. Rev. Lett. 78, 5010 (1997).

[9] C. Léger, J. Elezgaray, and F. Argoul, Phys. Rev. E 58, 7700 (1998).

[10] Y. Sawada, A. Dougherty, and J. P. Gollub, Phys. Rev. Lett. 56, 1260 (1986).

[11] P. P. Trigueros, J. Claret, F. Mas, and F. Sagués, J. Electroanal. Chem. Interfacial Electrochem. 312, 219 (1991).

[12] T. A. Witten and L. M. Sander, Phys. Rev. Lett. 47, 1400 (1981).

[13] T. Vicsek, Phys. Rev. Lett. 53, 2281 (1984).

[14] F. Argoul, A. Arneodo, G. Grasseau, and H. L. Swinney, Phys. Rev. Lett. 61, 2558 (1988).

[15] R.-F. Xiao, J. I. D. Alexander, and F. Rosenberg, Phys. Rev. A 38, 2447 (1988).

[16] A. Sánchez, M. J. Bernal, and J. M. Riveiro, Phys. Rev. E 50, R2427 (1994).

[17] M.-Q. López-Salvans, F. Sagués, J. Claret, and J. Bassas, Phys. Rev. E 56, 6869 (1997), and references therein.

[18] M.-Q. López-Salvans, P. P. Trigueros, S. Vallmitjana, J. Claret, and F. Sagués, Phys. Rev. Lett. 76, 4062 (1996).

[19] J. M. Pastor and M. A. Rubio, Phys. Rev. Lett. 76, 1848 (1996).

[20] J. M. Pastor, Ph.D. thesis, Universidad Nacional de Educación a Distancia (Spain), 1997.

[21] G. L. M. K. S. Kahanda, X.-Q. Zhou, R. Farrell, and P.-Z. Wong, Phys. Rev. Lett. 68, 3741 (1992).

[22] A. Iwamoto, T. Yoshinobu, and H. Iwasaki, Phys. Rev. Lett. 72, 4025 (1994).

[23] M. Castro, R. Cuerno, A. Sánchez, and F. Domínguez-Adame, Phys. Rev. E 57, R2491 (1998).

[24] P. L. Schilardi, O. Azzaroni, R. C. Salvarezza, and A. J. Arvia, Phys. Rev. B 59, 4638 (1999).

[25] T. A. Witten and L. M. Sander, Phys. Rev. B 27, 5686 (1983).

[26] M. Nauenberg, R. Richter, and L. M. Sander, Phys. Rev. B 28, 1649 (1983).

[27] P. Keblinski, A. Maritan, F. Toigo, R. Messier, and J. R. Banavar, Phys. Rev. E 53, 759 (1996).

[28] J. R. Banavar, M. Kohmoto, and J. Roberts, Phys. Rev. A 33, 2065 (1986).

[29] R.-F. Xiao, J. I. Alexander, and F. Rosenberger, Phys. Rev. A 39, 6397 (1989).

[30] J. Erlebacher, P. C. Searson, and K. Sieradzki, Phys. Rev. Lett. 71, 3311 (1993).

[31] R. F. Voss, Phys. Rev. B 30, 334 (1984); J. Stat. Phys. 36, 861 (1984).

[32] M. Uwaha and Y. Saito, Phys. Rev. A 40, 4716 (1989).

[33] T. Nagatani and F. Sagués, Phys. Rev. A 44, 6723 (1991); 44, 8303 (1991); T. Nagatani, ibid. 46, 2022 (1992).

[34] P. Meakin, Phys. Rev. B 28, 5221 (1983).

[35] J. M. Riveiro and M. J. Bernal, J. Non-Cryst. Solids 160, 18 (1993).

[36] J. Huth, H. Swinney, W. McCormick, A. Kuhn, and F. Argoul, Phys. Rev. E 51, 3444 (1995).

[37] J.-N. Chazalviel, M. Rosso, E. Chassaing, and V. Fleury, J. Electroanal. Chem. 407, 61 (1996).

[38] W. W. Mullins, J. Appl. Phys. 28, 333 (1957).
[39] Our model does ignore the evolution of the anions, which is coupled with that of the cations, assuming electroneutrality. This assumption is strictly correct all along the cell except in the neighborhood of the aggregate, the so called depletion zone, or space charge region. The width $l$ of this region depends on the applied voltage $V$ as $l \sim V^{2 / 3}$ [see J.-N. Chazalviel, Phys. Rev. A 42, 7355 (1990)]. For the experiments we have compared to or just referred to in our paper, this region ranges from 10 to $100 \mu \mathrm{m}$. Comparing the maximum value (100 $\mu \mathrm{m})$ with the characteristic lateral width of the resulting branches (the order of magnitude of this width is $1 \mathrm{~mm}$ ), we find that these branches are five times wider than the depletion zone. Translating that into the units of our model we see that for large applied electric field simulations (large $p$ values, in terms of our model), the resulting branch width (of about 50 lattice spacings) gives a depletion zone of about 10 lattice spacings (consistent, as it should be, with the profiles shown in Fig. 2). Thus, for small applied voltages the model is accurate enough, although in the worst case (we have assumed above the worst scenario) some discrepancies might appear for large voltages. However, in this case we expect that due to the large value of $p$ (the current) and the absence of particles next to the aggregate, cations are driven ballistically to it.

[40] W. Huang and D. B. Hibbert, Physica A 233, 888 (1996); a similar model is studied by J. Heinonen, I. Bukharev, T. AlaNissila, and J. M. Kosterlitz, Phys. Rev. E 57, 6851 (1998) in the context of step flow dynamics.

[41] At high concentration values, the diffusion coefficient $D_{c}$ and the mobility $\mu_{c}$ depend locally on the concentration field $C(\mathbf{x}, t)$, as C. Léger et al. have reported [8].

[42] We stress that even if the probabilities are different from those in Refs. [16] and [23], the drift velocity toward the cathode can be shown in all cases to be proportional to $p$.

[43] M. Castro and R. Cuerno (unpublished).

[44] H. Yan, Phys. Rev. Lett. 68, 3048 (1992).

[45] B. D. Hughes, Random Walks and Random Environments (Oxford University Press, New York, 1995), Vol. 1.

[46] See the review by J. Krug, Adv. Phys. 46, 139 (1997).

[47] S. Das Sarma, C. J. Lanczycki, S. V. Ghaisas, and J. M. Kim, Phys. Rev. B 49, 10693 (1994).

[48] M. Schimschak and J. Krug, Phys. Rev. B 52, 8550 (1995).

[49] F. Family and T. Vicsek, J. Phys. A 18, L75 (1985).

[50] M. Schroeder, M. Siegert, D. E. Wolf, J. D. Shore, and M. Plischke, Europhys. Lett. 24, 563 (1993).

[51] J. M. López and M. A. Rodríguez, Phys. Rev. E 54, R2189 (1996).

[52] J. M. López, M. A. Rodríguez, and R. Cuerno, Phys. Rev. E 56, 3993 (1997); Physica A 246, 329 (1997).

[53] T. Vicsek, Fractal Growth Phenomena, 2nd ed. (World Scientific, Singapore, 1992).

[54] P. Keblinski, Phys. Rev. Lett. 71, 805 (1993).

[55] G. L. M. K. S. Kahanda and P.-Z. Wong, Phys. Rev. Lett. 71, 806 (1993).

[56] M. Kardar, G. Parisi, and Y.-C. Zhang, Phys. Rev. Lett. 56, 889 (1986).

[57] S. F. Edwards and D. R. Wilkinson, Proc. R. Soc. London, Ser. A 381, 17 (1982).

[58] L. Vázquez, R. C. Salvarezza, and A. J. Arvia, Phys. Rev. Lett. 79, 709 (1997). 
[59] J. Villain, J. Phys. I 1, 19 (1991); Z.-W. Lai and S. Das Sarma, Phys. Rev. Lett. 66, 2348 (1991).

[60] The behavior of $S(k, t)$ at high $k$ in Fig. 15 cannot be explained as a signature of intrinsic anomalous scaling [Eq. (10)] either, since this would lead to an unphysical value $\alpha_{l o c}>1$; see H. Leschhorn and L.-H. Tang, Phys. Rev. Lett. 70, 2973 (1993).

[61] F. Family and J. G. Amar, Fractals 1, 753 (1993). 